\title{
Cause-specific telomere factors deregulation in hepatocellular carcinoma
}

Manale El Idrissi ${ }^{1}$, Valérie Hervieu ${ }^{2}$, Philippe Merle ${ }^{3,4,5}$, Franck Mortreux ${ }^{1}$ and Eric Wattel ${ }^{1,6^{*}}$

\begin{abstract}
Background: Among the numerous genetic defects associated with hepatocarcinogenesis, telomere abnormalities appear to play a role both in tumor promotion and maintenance. Telomeres, the chromosome extremities, are protected by specific proteins, the shelterin complex and by additional factors. Besides telomerase dysregulation, expression changes of these telomere factors have been observed in cancers.
\end{abstract}

Methods: Here, we tested the hypothesis that such dysregulation might occur in hepatocellular carcinoma (HCC) with specific patterns depending on the cause of HCC. We compared telomere length, telomerase activity (TA), hTERT and telomere genes expression using PCR and Western-blot analyses between non-cirrhotic liver, peritumoral cirrhotic tissue (40 samples) and cancerous tissue (40 samples) derived from 40 patients with HBV-, HCV-, or alcohol-related HCC.

Results: Alterations in TA, hTERT expression and telomere length between non-cirrhotic, cirrhotic, and tumor samples were not significantly influenced by the cause of HCC. In contrast, the expression pattern of $h T R$, shelterin, and nonshelterin telomere protective factors clearly distinguished the 3 causes of cirrhosis and HCC. For patients with HBV diseased liver, when compared with non-cirrhotic liver, the cirrhotic tissue underexpressed all shelterin and all but HMRE11A and RAD50 non-shelterin telomere factors. For HCV the expression level of POT1, RAP1, Ku80, and RAD50 was higher in cirrhotic than in non-cirrhotic liver samples without evidence for significant transcriptional change for the remaining genes. For alcohol-related liver diseases, the expression level of POT1, RAP1, TIN2, hMRE11A, hMRE11B, KU70, Ku80, RAD50, TANK1, and PINX1 was higher in cirrhotic than in non-cirrhotic liver samples. For the 3 causes of HCC, there was no significant change in shelterin and non-shelterin gene expression between cirrhosis and HCC samples.

Conclusions: These results validate our hypotheses and demonstrate that cirrhosis and HCC add-up numerous telomere dysfunctions including numerous cause-specific changes that appear to occur early during the course of the disease.

Keywords: Liver, Hepatocellular carcinoma, Telomere, Telomerase, Shelterin, Hepatitis B virus, Hepatitis C virus, Alcohol, Cirrhosis

\section{Background}

Hepatitis B (HBV) or C virus (HCV) infection and alcohol consumption are leading causes of hepatocellular carcinoma (HCC) that predominantly develops from chronic hepatitis and cirrhosis [1]. Among the numerous genetic and epigenetic defects associated with carcinogenesis [2], telomere abnormalities play a role in tumor promotion and maintenance [3-9]. Telomeres, the chromosome

\footnotetext{
* Correspondence: eric.wattel@ens-lyon.fr

'UMR5239 Oncovirologie et Biothérapies, Faculté de Médecine Lyon Sud, ENS - HCL, Université Lyon 1, CNRS, Pierre Bénite, France

${ }^{6}$ Service d'Hématologie, Pavillon Marcel Bérard, Centre Hospitalier Lyon-Sud, Université Lyon I, 165, chemin du Grand Revoyet, 69495 Pierre Bénite, France Full list of author information is available at the end of the article
}

extremities, are elongated by the human telomerase, the catalytic moiety of which is encoded by the human telomerase reverse transcriptase (hTERT) gene [10]. Additionally, telomeres are protected by specific proteins, the shelterin complex [11] and by additional non-specific factors such as human meiotic recombination 11 homolog A and B (hMRE11A and B), Ku proteins 70 and 80 (Ku70 and Ku80), Nijmegen breakage syndrome-1 (NBS1), RAD50, tankyrase 1 and 2 (TANK1 and 2), Werner syndrome helicase (WRN), and PIN2/TRF1-interacting, telomerase inhibitor 1 (PINX1) [12]. These factors prevent telomere degradation and facilitate telomerase-based telomere elongation.

\section{Biomed Central}


Short or unprotected telomeres are recombinogenic and can therefore promote tumorigenesis [3]. In normal cells, dysfunctional telomeres trigger the DNA damage response and replicative cellular senescence [10,13-18]. Early oncogenic events frequently involve evasion of the DNA damage response, which allows the clonal persistence of cells bearing a telomere-associated genetic instability. During early tumor development, hTERT is frequently expressed and allows the clone to bypass mitotic catastrophe and replicative senescence, contributing to malignant immortalization [4,5,19-21]. Therefore, impaired telomere protection and/or elongation represent putative oncogenic events. Indeed, numerous oncogenes or tumor suppressor genes have been reported to interfere with the telomere machinery. In the liver, telomere shortening correlates with chromosomal instability and the development of HCC $[4,6,8]$. Hepatotropic viruses and alcohol have been reported to interfere with telomere homeostasis. For example, $h T E R T$ transcription was found to be activated upon HBV DNA integration in the vicinity of the $h T E R T$ gene [22] while HBV encoded X (HBx) [23-27] or preS2 [28,29] proteins promote hTERT expression and contributed to clonal persistence. However, some mutated HBx have been reported to possess repressive effects on hTERT transcription [25]. The HCV core protein has been demonstrated to enhance telomerase activity [30] while alcohol exposure triggers premature senescence with accelerated telomere shortening [31].

Changes in telomere length, telomerase activity and hTERT expression have been extensively explored at different steps of hepatocarcinogenesis. However, to our knowledge, the status of shelterin and non-shelterin telomere factors has not been examined during liver carcinogenesis. Furthermore, little is known about the interactions between telomere alterations and the cause of HCC, although hepatitis viruses and alcohol are known to possess specific and distinct effect on telomere homeostasis in vitro [22,23,25-29,31]. Dissecting telomere factors' deregulation during carcinogenesis has revealed novel oncogenic pathways, prognostic markers, and therapeutic targets aimed at preventing or treating cancers. The aim of this study was to determine whether the main causes of HCC might trigger distinct telomere effects in vivo.

\section{Methods}

\section{Samples}

The medical ethics committee of the Hospices Civils de Lyon approved the study, and the informed consent was obtained from patients in accordance with the Declaration of Helsinki and with institutional guidelines. The study population consisted of paired tumor and non-tumor tissues from 40 patients suffering from $\mathrm{HCC}$ including 10 HBV-, $10 \mathrm{HCV}-, 10$ alcohol-related and $10 \mathrm{HCC}$ without $\mathrm{HCV}$ viral or alcohol exposure (idiopathic HCC).
Serological markers for HBV (HBsAg, HBeAg and anti$\mathrm{HBe}$ ), HCV (anti-HCV) were tested in all cases by commercial enzyme immunoassays. The clinical and histological data accompanying the samples analyzed are shown in Table 1. Tissue samples from patients resected for $\mathrm{HCC}$ were collected during surgery and divided into two parts: one was immediately cut into small pieces, snap-frozen in liquid nitrogen and stored in deep freezer; the other was fixed in $10 \%$ formalin and paraffin-embedded for histopathological examination and immunohistochemistry. Histological analysis and immunohistochemistry were performed at the Department of Pathology at the Hospices Civils de Lyon.

\section{Telomere length assay}

Each sample was homogenized and total cellular DNA was extracted using phenol chloroform. The average telomere length was measured in all samples using the TeloTAGGG Telomere length Assay (Roche). Briefly, purified genomic DNA $(6-8 \mu \mathrm{g})$ was digested by specific restriction enzymes. The DNA fragments were separated by gel electrophoresis and transferred to a nylon membrane using Southern blotting. The blotted DNA fragments were hybridized to a digoxigenin-labeled probe specific to telomere repeats and incubated with a digoxigenin-specific antibody coupled to alkaline phosphate. Finally, the immobilized probe was visualized by a sensitive chemiluminescence substrate and the average TRF length was assessed by comparing the signals relative to a molecular weight standard.

\section{Quantification of telomerase activity}

The telomeric repeats amplification protocol (TRAP) was combined with real-time detection of amplification products to determine telomerase activity using a Quantitative Telomerase Detection kit (US Biomax) following the manufacturer's recommendations. Total protein extracts $(0.5 \mu \mathrm{g})$ were used for each reaction. The end products were resolved by PAGE on a $12.5 \%$ non-denaturing gel, stained with Sybr Green Nucleic Acid gel stain (Invitrogen) and visualized using the Bio-Rad Molecular Imager ChemiDoc System.

\section{Real-time quantitative reverse transcriptase-polymerase chain reaction (PCR)}

Each tissue sample was homogenized and total cellular RNA was extracted using the MasterPure Complete DNA and RNA Purification Kit (Epicentre) according to the manufacturer's instructions. Before reverse transcription, RNA was treated with DNase (Invitrogen-Life technology) to prevent DNA contamination. First-strand complementary DNA (cDNA) was synthesized from $0.5 \mu \mathrm{g}$ RNA using random primers (Promega) and Superscript II reverse transcriptase (Invitrogen). The RNA concentration and purity were determined using a NanoDrop instrument 
immunohistochemistry was quantified by a pathologist. The percentage of labeled nuclear area over the total neoplastic and the non-neoplastic nuclear area in the section was quantified from 2000 cells in areas of highest nuclear labeling.

\section{Statistical analysis}

Statistical analysis was performed using the 2-tailed Student's t test or the Mann-Whitney U rank sum test. $\mathrm{P}<0.05$ was considered statistically significant in all analyses. All data analyses were performed using SPSS statistical software version 20 .

\section{Results}

The main objective of this study was to determine whether differences exist in telomere deregulation between HBV-, $\mathrm{HCV}-$, and alcohol-associated liver carcinogenesis. Liver carcinogenesis is a multistep process where clinical and histopathological features frequently permits the differentiation of the two main phases that include a cirrhotic stage followed by the development of overt HCC. Our collection of 80 liver samples was obtained from 40 patients with HCC. For each case 2 samples were analyzed that corresponded to tumoral and peritumoral tissue. The Table 1 shows that in 12 cases of HCC, peritumoral samples corresponded to histologically normal, non-cirrhotic liver tissue whereas in the 28 remaining cases, the peritumoral tissue was cirrhotic. We assumed that the development of cirrhosis from a histologically non-cirrhotic liver represents an early event during liver carcinogenesis, whereas the development of HCC from a cirrhotic liver reflects later carcinogenic events. Accordingly, in order to assess telomere dysregulation at the early and late stages of liver carcinogenesis, we compared cell proliferation, Telomere Restriction Fragment (TRF) length, TA, hTERT, human telomerase RNA compound ( $h T R$ ), shelterin and non-shelterin telomere factor expression between noncirrhotic and cirrhotic liver samples and between cirrhotic and HCC liver samples for each cause of HCC (Additional file 1: Table S1, Additional file 2: Table S2. Figure 1 represents the distribution of TRF length, hTERT and $h T R$ expression, TA (Figure 1A) and telomere factors expression (Figure 1B) in peritumoral and tumoral samples derived from patients suffering from idiopathic, $\mathrm{HBV}_{-}, \mathrm{HCV}-$, and alcohol-related HCC. Figure 2 represents the expression of Ki67 (Figure 2A), hTERT (Figure 2B) and telomere protective factors (Figure $2 \mathrm{~B}$ and $\mathrm{C}$ ) at the protein level.

\section{Telomere deregulation at the early stage of HBV-associated hepatocarcinogenesis}

Expression of the proliferative marker Ki67 was not significantly different between the $8 \mathrm{HBV}$ positive cirrhotic samples and the 12 non-cirrhotic liver samples deriving from patients with HCC. As illustrated in Figure 1A, the level of $h T E R T$ expression was significantly higher in the $8 \mathrm{HBV}$ positive cirrhotic samples than in the 12 noncirrhotic liver samples $(\mathrm{p}=0.040$, Mann-Whitney test). In contrast, there was no significant difference in the level of TA between the cirrhotic and non-cirrhotic sample categories. HBV-associated cirrhosis expressed significantly lower $h T R$ levels when compared to histologically non-cirrhotic liver tissue: 0.0053 versus 0.3574 arbitrary units ( $\mathrm{p}<10^{-4}$, Mann-Whitney test) (Figure 1A). The TRF length was longer in HBV positive cirrhotic samples than in non-cirrhotic samples (6.60 kbp versus $5.69 \mathrm{kbp}$ ) but the difference was not statistically significant. Comparative Western-blot analysis of hTERT expression in HBV positive cirrhotic samples versus non-cirrhotic liver samples confirmed the qRTPCR results for hTERT expression (Figure 2B). Table 2 and Figure $1 \mathrm{~B}$ show that all shelterin and non-shelterin telomere factors except HMRE11A and RAD50 were significantly underexpressed in HBV positive peritumoral cirrhotic samples. Comparative Western-blot analysis confirmed that protection of telomeres 1 (POT1), telomere repeat factor 2 (TRF2), HMR11A/B, and Ku80 had lower expression levels in $\mathrm{HBV}$ positive cirrhotic samples than in non-cirrhotic liver samples (Figure $2 \mathrm{C}$ and D). These results suggest that at the telomere level, the development of HBV-associated cirrhosis includes strong $h T E R T$ overexpression and considerable repression of $h T R$, shelterin, and non-shelterin telomere factors. Similar results were obtained when the $8 \mathrm{HBV}^{+}$ cirrhotic samples were compared with the 9 noncirrhotic liver samples derived from patients with idiopathic HCC (data not shown).

\section{Telomere deregulation at the early stage of HCV-associated hepatocarcinogenesis}

Expression of the Ki67 proliferation marker was not significantly different between the $9 \mathrm{HCV}$ positive cirrhotic samples and the 12 non-cirrhotic liver samples deriving from patients with HCC. There was no significant difference in the expression level of TA, $h T E R T$ and $h T R$ between the two sample categories (Figure 1A). Western-blot analysis of hTERT expression confirmed the qRTPCR results for $h T E R T$ expression (Figure 2B). Shelterin, POT1 and repressor-activator protein 1 (RAP1) were demonstrated to be significantly overexpressed in $\mathrm{HCV}$ positive cirrhotic samples when compared with noncirrhotic liver samples. The remaining factors displayed an identical $(T R F 2)$ or a non-significant reduced expression level (Table 2). In contrast to HBV, all telomere factors except Pinx1 non-shelterin were overexpressed in cirrhotic peritumoral $\mathrm{HCV}$ positive samples, as compared to noncirrhotic liver samples (Figure $1 C$, Table 2). Indeed, the expression of $K u 80(\mathrm{p}=0.029)$ and $R A D 50(\mathrm{p}=0.018)$ was approximately 3 times higher than that of the control samples. Western-blots confirmed that POT1, HMRE11A/B, 

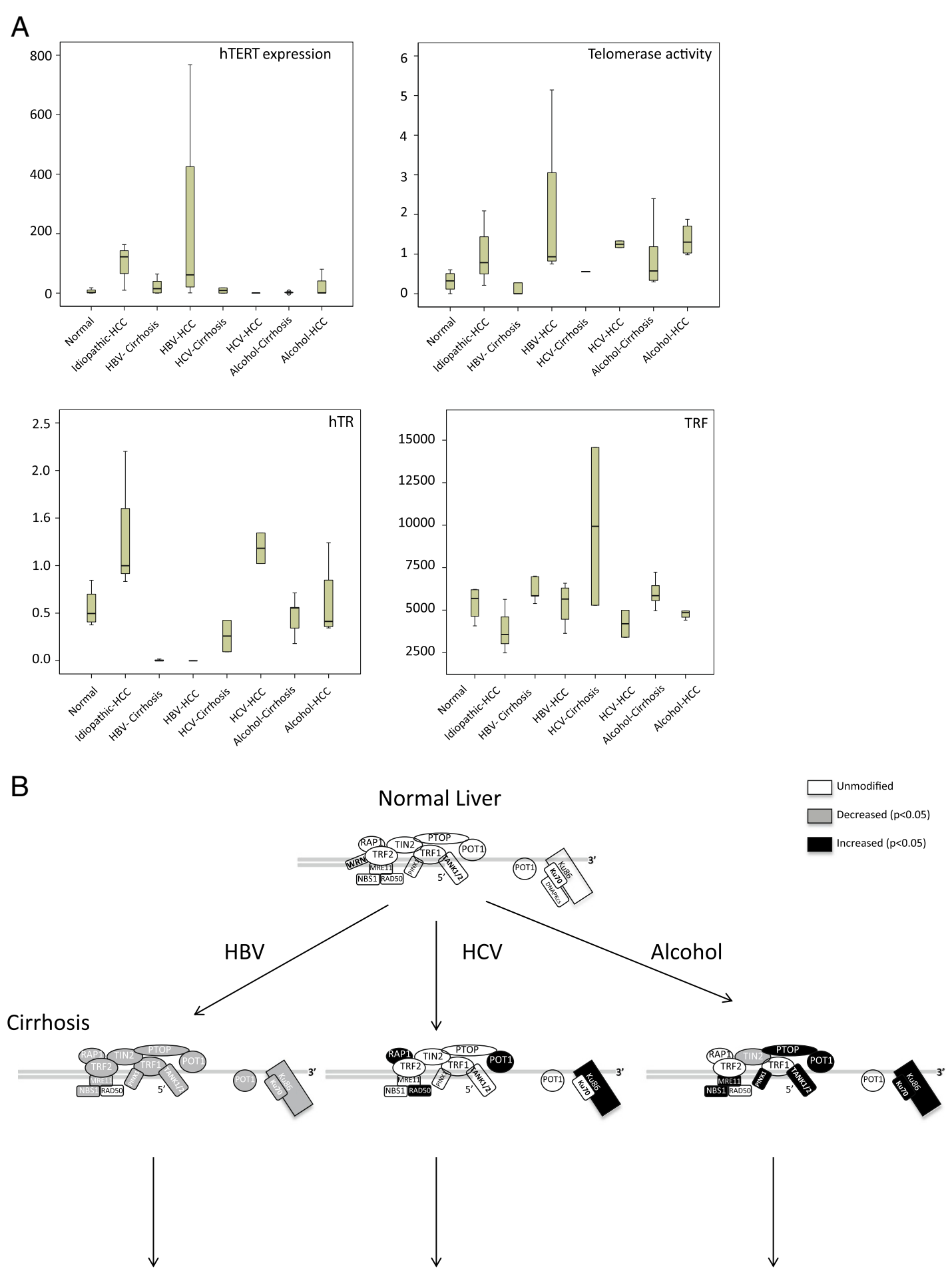

$\mathrm{HCC}$

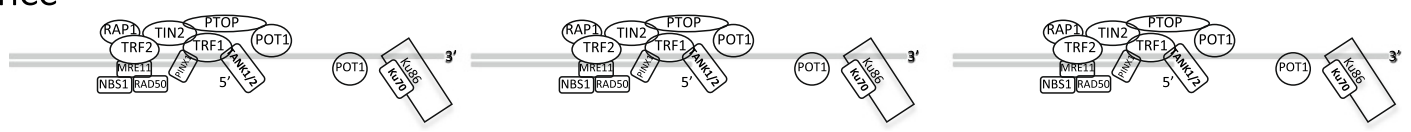

Figure 1 (See legend on next page.) 
(See figure on previous page.)

Figure 1 Common and specific telomere abnormalities between HBV-, HCV-, and alcohol-associated cirrhosis and hepatocellular carcinoma. A. Distribution of hTERT and hTER expression, telomerase activity and TRF length among the main causes of hepatocellular carcinoma. B. Alteration in shelterin and non-shelterin gene expression at the two main steps of liver carcinogenesis in vivo. Significantly overexpressed genes ( $p<0.05$, Mann Whitney test) are represented in black whereas significantly underexpressed genes are represented in gray.

and KU80 were more expressed in HCV positive cirrhotic samples than in non-cirrhotic liver samples (Figure 2D). These results suggested that at the telomere level, the main changes that accompany the development of $\mathrm{HCV}$-associated cirrhosis predominately involve the overexpression of POT1, RAP1, Ku80, and RAD50 telomere factors.
Telomere deregulation at the early stage of alcohol-associated hepatocarcinogenesis

Expression of the Ki67 proliferative marker was not significantly different between alcohol-associated cirrhotic and non-cirrhotic liver tissues deriving from patients with HCC. There was no significant difference in TRF length, TA, $h T E R T$ and $h T R$ expression between the two sample

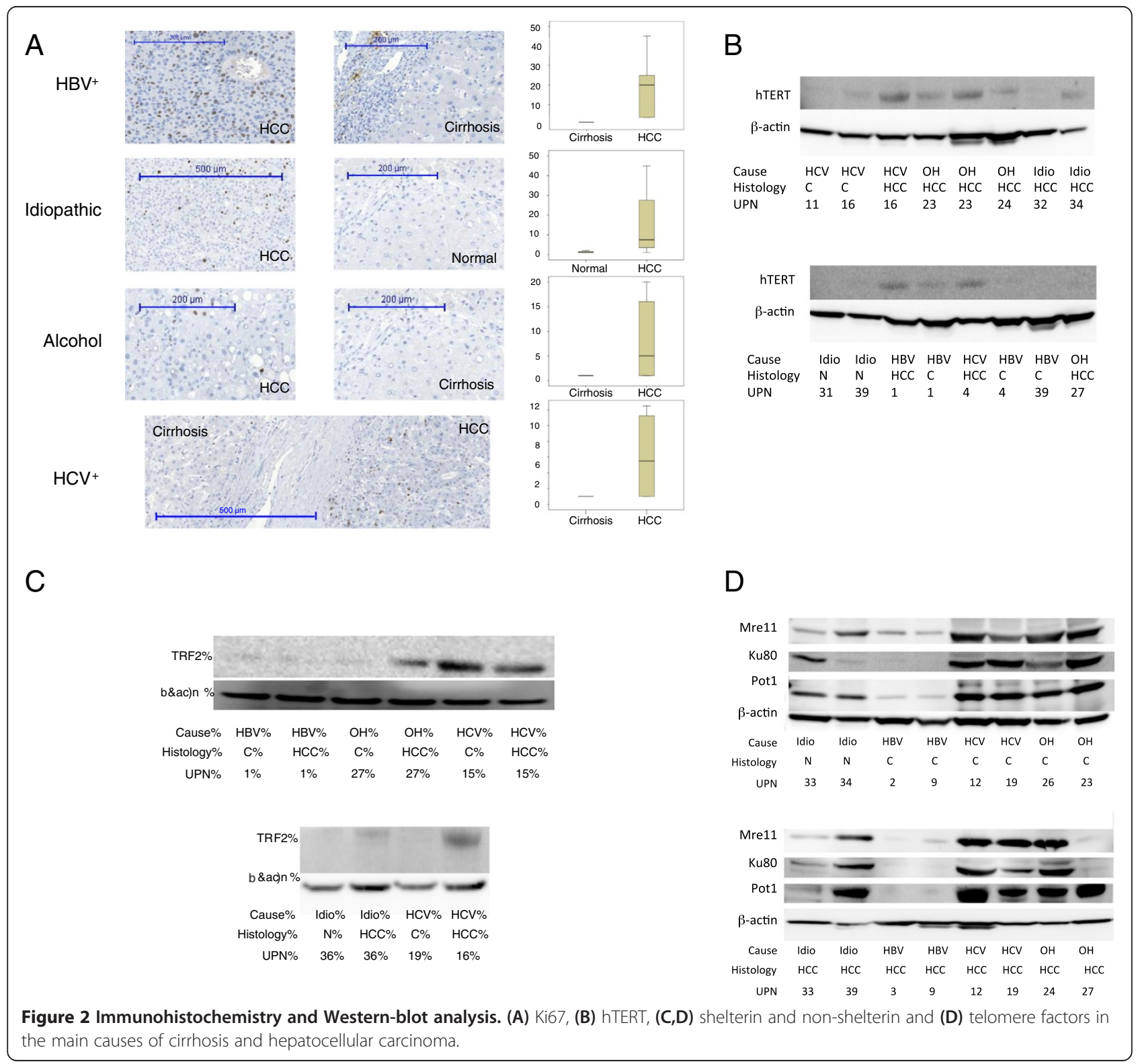


Table 2 Cause-specific differences in telomeric gene expression between cirrhotic and non-cirrhotic liver samples

\begin{tabular}{|c|c|c|c|c|c|c|c|}
\hline & \multirow{2}{*}{$\begin{array}{l}\text { Non-cirrhotic } \\
(n=12)\end{array}$} & \multicolumn{3}{|c|}{ Cirrhotic } & \multicolumn{3}{|c|}{$p$} \\
\hline & & $\mathrm{HBV}(n=8)$ & $\mathrm{HCV}(n=9)$ & Alcohol $(n=10)$ & For HBV & For $\mathrm{HCV}$ & For alcohol \\
\hline \multicolumn{8}{|l|}{ Shelterin } \\
\hline POT1 & 0.0021 & 0.0000 & 0.0125 & 0.0090 & 0.0480 & 0.0100 & 0.0050 \\
\hline PTOP & 0.0094 & 0.0000 & 0.0037 & 0.0055 & 0.0200 & ns & ns \\
\hline RAP1 & 0.1570 & 0.0016 & 0.4210 & 0.4091 & 0.0070 & 0.0080 & 0.0060 \\
\hline TIN2 & 0.3510 & 0.0018 & 0.0510 & 0.0804 & 0.0010 & ns & $<10-4$ \\
\hline TRF1 & 0.5585 & 0.0117 & 0.2271 & 0.2488 & $<10-4$ & ns & ns \\
\hline TRF2 & 0.0016 & 0.0000 & 0.0016 & 0.0012 & 0.0050 & ns & ns \\
\hline \multicolumn{8}{|c|}{ Non-Shelterin } \\
\hline HMRE11A & 0.0187 & 0.0006 & 0.0627 & 0.0764 & ns & ns & 0.0070 \\
\hline HMRE $11 B$ & 0.0359 & 0.0008 & 0.0492 & 0.0886 & 0.0030 & ns & 0.0020 \\
\hline Ku>0 & 0.0955 & 0.0045 & 0.1704 & 0.1825 & $<10-4$ & ns & 0.0440 \\
\hline Kuso & 0.0408 & 0.0033 & 0.1209 & 0.1316 & 0.0200 & 0.0290 & 0.0120 \\
\hline NBS1 & 0.0266 & 0.0002 & 0.0304 & 0.0403 & 0.0030 & ns & ns \\
\hline RAD50 & 0.0030 & 0.0002 & 0.0091 & 0.0108 & ns & 0.0180 & 0.0500 \\
\hline TANK1 & 0.0468 & 0.0005 & 0.0788 & 0.0945 & $<10-4$ & ns & 0.0030 \\
\hline TANK2 & 0.0129 & 0.0000 & 0.0188 & 0.0127 & 0.0200 & ns & ns \\
\hline Pin $\times 1$ & 0.0131 & 0.0001 & 0.0083 & 0.0219 & 0.0020 & ns & 0.0210 \\
\hline
\end{tabular}

categories (Figure 1A). Western-blot analysis of hTERT expression confirmed the qRTPCR results (Figure $2 \mathrm{~B}$ ). Shelterin, POT1 ( $\mathrm{p}=0.005)$ and RAP1 ( $\mathrm{p}=0.006)$ were demonstrated to be significantly overexpressed in alcoholassociated cirrhotic tissues, whereas other shelterins were found to be underexpressed, with TRF1-interacting nuclear protein 2 gene (TIN2) showing a significant difference (Table 2). All non-shelterin telomere factors, except TANK2 and Pinx1, contained a transcriptional pattern that resembled that in HCV cirrhotic samples. Accordingly, all telomere factors except the TANK2 non-shelterin were overexpressed in cirrhotic alcoholexposed liver with significant differences demonstrated for HMRE11A, HMRE11B, Ku70, Ku80, RAD50, TANK1, and Pinx1 (Table 2, Figure 1C). Western-blot analyses confirmed the qRTPCR results for POT1, TRF2, HMR11A/B, and KU80 (Figure $2 \mathrm{C}$ and D). These results suggested that at the telomere level, the main changes accompanying the development of alcohol-associated cirrhosis and fibrosis predominantly involve the overexpression of POT1, RAP1, HMRE11A, HMRE11B, Ku70, Ku80, RAD50, TANK1, and Pinx 1 telomere factors. Taken together, these results indicate that the development of HBV-, $\mathrm{HCV}_{-}$, and alcohol-related cirrhosis rely on clearly distinct telomere perturbations and suggests that these distinct carcinogens possess specific effects on telomere homeostasis. Consequently, 3 kinds of cirrhotic tissues displayed significant differences in the expression of telomere factors (Figure 1, Additional file 3: Table S3).
Telomere deregulation at the late stage of

\section{HBV-associated hepatocarcinogenesis}

Having demonstrated the cause-specific changes in telomere factors' expression between cirrhotic and noncirrhotic livers, i.e. during early hepatocarcinogenesis, we next sought to investigate whether these differences persist at the late stages of HCC development. To this end we compared telomere deregulations between cirrhotic and tumoral samples deriving from patients with HCC. We first compared the $10 \mathrm{HBV}$-associated HCC samples with their 8 cirrhotic peritumoral samples. Expression of the Ki67 proliferative marker was significantly increased in HBV-associated HCC, as compared with HBV-associated cirrhosis ( $\mathrm{p}=0.002$, Mann-Whitney test). The TRF length was significantly shorter in tumor samples than in cirrhotic samples $(\mathrm{p}=0.05$, MannWhitney test) whereas the levels of TA and hTERT expression were significantly higher in HBV positive HCC ( $\mathrm{p}=0.017$ for hTERT and $\mathrm{p}=0.002$ for TA, MannWhitney test) without any significant difference in the level of $h T R$ expression between the 2 tissues (Figure 1A). Western-blotting analyses confirmed the qRTPCR results for hTERT expression (Figure 2B). Table 3 shows that with the exception of $\operatorname{Pin} x 1$, where there was a trend for higher expression in HCC, all shelterin and non-shelterin genes remained underexpressed in $\mathrm{HBV}$ positive $\mathrm{HCC}$ without any significant difference between cirrhosis and HCC. Western-blot analysis of TRF2, HMRE11A/B, Ku80, and POT1 confirmed the qRTPCR results (Figure 2C and D). 
Table 3 Cause-specific differences in telomeric gene expression between cirrhotic/fibrotic and HCC tissue samples

\begin{tabular}{|c|c|c|c|c|c|c|c|c|c|}
\hline & \multicolumn{3}{|c|}{ HBV } & \multicolumn{3}{|c|}{$\mathrm{HCV}$} & \multicolumn{2}{|l|}{ Alcohol } & \multirow[b]{2}{*}{$p$} \\
\hline & $\begin{array}{l}\text { Cirrhotic and/or } \\
\text { Fibrotic }(n=8)\end{array}$ & $\mathrm{HCC}(n=10)$ & $p$ & $\begin{array}{l}\text { Cirrhotic and/or } \\
\text { Fibrotic }(n=9)\end{array}$ & $\mathrm{HCC}(n=10)$ & $p$ & $\begin{array}{l}\text { Cirrhotic and/or } \\
\text { Fibrotic }(n=10)\end{array}$ & HCC $(n=10)$ & \\
\hline \multicolumn{10}{|l|}{ Shelterin } \\
\hline POT1 & 0.0000 & 0.0000 & ns & 0.0125 & 0.0203 & ns & 0.0090 & 0.0060 & ns \\
\hline PTOP & 0.0000 & 0.0000 & ns & 0.0037 & 0.0064 & ns & 0.0055 & 0.0071 & ns \\
\hline RAP1 & 0.0016 & 0.0000 & ns & 0.4210 & 0.5059 & ns & 0.4091 & 0.2538 & ns \\
\hline TIN2 & 0.0018 & 0.0033 & ns & 0.0510 & 0.0581 & ns & 0.0804 & 0.0876 & ns \\
\hline TRF1 & 0.0117 & 0.0209 & ns & 0.2271 & 0.1626 & ns & 0.2488 & 0.2886 & ns \\
\hline TRF2 & 0.0000 & 0.0000 & ns & 0.0061 & 0.0015 & ns & 0.0012 & 0.0012 & ns \\
\hline \multicolumn{10}{|c|}{ Non Shelterin } \\
\hline HMREI1A & 0.0006 & 0.0000 & ns & 0.0627 & 0.0811 & ns & 0.0764 & 0.0536 & ns \\
\hline HMRE11B & 0.0008 & 0.0000 & ns & 0.0492 & 0.0508 & ns & 0.0886 & 0.0850 & ns \\
\hline Ku>0 & 0.0045 & 0.0024 & ns & 0.1704 & 0.2418 & ns & 0.1825 & 0.1645 & ns \\
\hline Ku80 & 0.0033 & 0.0015 & ns & 0.1209 & 0.1494 & ns & 0.1316 & 0.0853 & ns \\
\hline NBS1 & 0.0002 & 0.0024 & ns & 0.0304 & 0.0317 & ns & 0.0403 & 0.0501 & ns \\
\hline RAD50 & 0.0002 & 0.0000 & ns & 0.0091 & 0.0118 & ns & 0.0108 & 0.0101 & ns \\
\hline TANK1 & 0.0005 & 0.0000 & ns & 0.0788 & 0.0761 & ns & 0.0945 & 0.0869 & ns \\
\hline TANK2 & 0.0000 & 0.0006 & ns & 0.0188 & 0.0255 & ns & 0.0127 & 0.0171 & ns \\
\hline Pin $x 1$ & 0.0001 & 0.0049 & ns (0.054) & 0.0083 & 0.0107 & ns & 0.0219 & 0.0165 & ns \\
\hline
\end{tabular}

These results suggested that at the telomere level, augmented TA and $h T E R T$ expression represent the major significant telomere deregulation distinguishing HBVassociated HCC from HBV-associated cirrhosis. Accordingly, comparison of HBV-related HCC with non-cirrhotic liver samples demonstrated similar differences as the comparison of HBV-related cirrhosis with non-cirrhotic liver samples (Additional file 4: Table S4).

\section{Telomere deregulation at the late stage of} HCV-associated hepatocarcinogenesis

$\mathrm{HCV}$-associated HCC expressed higher levels of the Ki67 proliferative marker (6\% versus $1 \%$ ) than peritumoral cirrhotic tissue samples but the difference was not statistically significant. When compared to their peritumoral cirrhotic tissue samples, $\mathrm{HCV}$ positive HCC expressed higher amounts of hTERT transcripts $(\mathrm{p}=0.54)$ and $h T R(\mathrm{p}=0.021)$ and they displayed increased TA $(\mathrm{p}=0.036)$ when compared with HCV positive cirrhosis (Figure 1A). The TRF length was shorter in HCV-associated cirrhosis than in HCC but the difference was not statistically significant (5.1 kbp versus 6.6 $\mathrm{kbp}, \mathrm{p}=0.39$ ) (Figure 1A). Table 3 shows that the pattern of shelterin and non-shelterin genes expression was not significantly different between $\mathrm{HCV}$-associated $\mathrm{HCC}$ and $\mathrm{HCV}$-associated cirrhosis. Western-blot analysis confirmed qRTPCR results (Figure $2 \mathrm{~B}, \mathrm{C}$, and D). These results suggested that at the telomere level, increased TA and $h T R$ expression represent the major significant telomere deregulation that distinguishes $\mathrm{HCV}$-associated $\mathrm{HCC}$ from $\mathrm{HCV}$-associated cirrhosis.

Telomere deregulation at the late stage of alcohol-associated hepatocarcinogenesis

When compared to their peritumoral cirrhotic tissue samples, alcohol-associated HCC expressed higher levels of the Ki67 proliferative marker ( $8 \%$ versus 1\%) but the difference was not statistically significant. Figure $1 \mathrm{~A}$ shows that TA, $h T E R T$ and $h T R$ expressions were augmented in alcohol-associated HCC but these differences were not statistically significant. Table 3 shows that the pattern of shelterin and non-shelterin genes expression was not significantly different between alcohol-associated HCC and alcohol-associated cirrhosis. Western-blot analysis confirmed the qRTPCR results (Figure $2 \mathrm{C}$ and $\mathrm{D}$ ). These results suggested that at the telomere level, there is no significant deregulation that distinguishes alcohol-associated HCC from alcohol-associated cirrhosis.

\section{Discussion}

The data suggest that the development of HCC involves the accumulation of numerous telomere dysfunctions that appear to include cause-specific deregulations. Our sample collection permitted the comparison of histologically non-cirrhotic livers with cirrhosis and HCC in the context of HBV and HCV infections, and alcohol exposure. Given that HCC mostly develop from cirrhotic 
livers, we assumed that comparing histologically noncirrhotic liver samples with cirrhotic liver samples would reflect early carcinogenesis whereas comparing cirrhotic liver samples with tumor samples would reflect later carcinogenic events. Indeed, alterations in TRF length, TA, $h T E R T$ and $h T R$ expression were identified at both the early and late steps of hepatocarcinogenesis. These alterations were observed roughly in parallel among the 3 different causes of HCC. In contrast, the numerous changes demonstrated in the expression of telomere protective factors appeared to be restricted to early hepatocarcinogenesis. Additionally, these changes permitted the identification of a gene expression signature for each cause of cirrhosis and HCC. There was furthermore, evidence that the telomere phenotype of HBV-associated-cirrhosis and HCC was different from that of the other causes of cirrhosis and HCC.

No correlation was found between TA, hTERT expression and telomere length with respect to the cause of cirrhosis and HCC. This result is in agreement with the study of Saini et al. who compared TA, TRF and hTERT expression between HBV, HCV, and non-B non-C-related HCC [34]. In contrast, Guo et al. reported that HbsAg positive $\mathrm{HCC}$ expressed higher amounts of hTERT mRNA than HbsAg negative HCC [35]. Whatever the cause, there was no significant difference in TRF length between cirrhotic and non-cirrhotic samples. This result was in agreement with the lack of difference in cell proliferation between the 2 samples categories, as assessed by the quantification of Ki67 expression. In contrast, the tumor samples expressed higher levels of the Ki67 proliferative marker and contained shorter telomeres than either non-cirrhotic or cirrhotic samples. There was no precise correlation between the level of $h T E R T$ expression measured by qRTPCR and the level of TA measured by the quantitative TRAP assay, suggesting that posttranscriptional modifications might participate to modulate TA during hepatocarcinogenesis. Additionally, there was no significant correlation between either $h T E R T$ expression or TA and telomere length. Conversely, Figure 1A shows that the shorter were the telomeres in sample sets, the higher were TA and $h T E R T$ expression in these samples. This conflicting data might be explained, at least in part, by changes in regulating access of the telomere to the telomerase in liver cells, i.e. by changes in telomere proteins content.

Accumulating evidence suggests that telomeric factors dysregulation is involved in cancer development as has been demonstrated in the maintenance of the tumor phenotype. To our knowledge, this study is the first which investigates the expression of the main telomere protective genes in the main subtype of cirrhosis and HCC. Previously, Oh et al. demonstrated that expression of TRF1, TRF2 and TIN2 was gradually increased according to the progression of hepatocarcinogenesis in $\mathrm{HBsAg}$ positive individuals [36]. In this study, HBV-, HCV- and alcoholassociated cirrhosis displayed significantly different distinct patterns of telomere protective factor expression, as compared with that of non-cirrhotic liver (Table 2). The 3 subtypes of cirrhosis possessed a specific signature, with respect to telomere protective factor expression (Additional file 3: Table S3). Although the expression level of all the shelterin and non-shelterin telomere factors was not equally distributed between the 3 causes of cirrhosis (Additional file 3: Table S3), the telomere phenotype of HBV-associated-cirrhosis appeared different from that of the 2 other causes of cirrhosis. When compared with noncirrhotic liver, HBV-associated cirrhosis displayed a dramatic repression of all shelterin and non-shelterin factors except HMRE11A and RAD50. In contrast, the alterations in telomere factor expression between non-cirrhotic and cirrhotic samples were similar between HCV- and alcoholassociated cirrhosis. Accordingly, the expression pattern of all telomere factors, except TIN2 and HMRE11B, was identical between HCV- and alcohol-associated cirrhosis (Additional file 3: Table S3). These results suggest that cause-specific factors are involved in initiating telomere dysfunction in the liver. For example, HBV-associated cirrhosis displayed very low amounts of TRF2 that has been demonstrated to elicit telomere shortening ex vivo [37].

Whatever the cause, the levels of shelterin and nonshelterin telomere factors expression were not significantly different between cirrhotic and HCC samples (Figure 1B and Table 3). Again, the expression pattern of telomere protective factor of $\mathrm{HBV}$-associated-HCC remained distant from that of the 2 other causes of HCC, which closely resembled that of idiopathic HCC (Additional file 5: Table S5). This suggests that in HCC, the cause-specific expression pattern of shelterin and non-shelterin factors has been acquired early during the course of the disease. Given that these factors are thought to prevent proper telomerase-telomere interaction, the present results partly explains the combination of high TA with short telomeres in HCC.

\section{Conclusion}

In conclusion, the control of telomere homeostasis is significantly dysregulated during liver carcinogenesis and each cause of cirrhosis and HCC includes specific dysregulation of telomere protective factors. These changes occur early, at the cirrhotic stage, and persist to the tumor stage, which suggests that they contribute to both tumor development and tumor progression. By demonstrating gene and protein dysregulation that are thought to prevent proper telomerase-telomere interactions, the present results partly explain the combination of high TA with short telomeres in HCC. Shortened and deprotected telomeres are recombinogenic and contribute to the genetic instability that characterize $\mathrm{HCC}$ and facilitate tumor progression, 
tumor recurrence and resistance to treatment [5-8,10]. Importantly, hepatocytes have been reported to tolerate telomere dysfunctions [37], reinforcing the tumorigenic impact of alcohol-, HBV-, and HCV-associated telomere damage in exposed individuals. Targeting telomerase is becoming a promising approach for the treatment of HCC [38-40] and our present results also support such an approach for treating the main causes of this disease. In contrast, our results suggest that targeting the cause-specific deregulation of telomere protective factors might be of interest in the prevention or the treatment of cirrhosis and HCC.

\section{Additional files}

Additional file 1: Table S1. Distribution of telomeric gene expression among the 12 non-cirrhotic and the 28 cirrhotic samples.

Additional file 2: Table S2. Distribution of telomeric gene expression among the 28 cirrhotic and the 40 tumor samples.

Additional file 3: Table S3. Distribution of telomeric gene expression among the $40 \mathrm{HCC}$ and the 12 non-cirrhotic liver samples.

Additional file 4: Table S4. Cause-specific distribution of telomere genes expression among the 28 cirrhotic liver samples.

Additional file 5: Table S5. Cause-specific distribution of telomere genes expression among the 40 HCC samples.

\section{Competing interests}

The authors declare that they have no competing interests.

\section{Authors' contributions}

MEI carried out the most experimental work. VH performed the sample collection and Ki67 assays. PM performed the sample collections, provided clinical data. PM, FM, and EW were responsible for the design of the study and its coordination. PM, EW, and FM wrote the manuscript. All authors read and approved the final manuscript.

\section{Acknowledgments}

This work was supported by the Lique Nationale contre le Cancer (comités de la Savoie, de la Loire et du Rhône), Agence Nationale pour la Recherche (ANR), Hospices Civils de Lyon, University Lyon I, Centre National pour la Recherche Scientifique (CNRS), and Institut National de la Santé et de la Recherche Médicale (Inserm). M.E.I. was supported by bursaries from the Région Rhône-Alpes (cluster 10) and from the Association pour la Recherche sur le Cancer (ARC). V.H. is supported by Hospices Civils de Lyon. C.K. is supported by the CNRS, P.M. is supported by Hospices Civils de Lyon and Lyon I University. F.M. is supported by Inserm and by Hospices Civils de Lyon (AVIESAN CHRT 2010). E.W. is supported by Hospices Civils de Lyon and Lyon I University.

\section{Author details}

'UMR5239 Oncovirologie et Biothérapies, Faculté de Médecine Lyon Sud, ENS - HCL, Université Lyon 1, CNRS, Pierre Bénite, France. ${ }^{2}$ Service Central d'Anatomie et Cytologie Pathologiques, Hospices Civils de Lyon, Université Lyon 1, Hôpital Edouard Herriot, 69437, Cedex 03 Lyon, France. ${ }^{3}$ INSERM U1052, CNRS UMR5286, Centre de Recherche en Cancérologie de Lyon, F69008 Lyon, France. ${ }^{4}$ Université Lyon-1, F69622 Villeurbanne, France. ${ }^{5}$ Hospices Civils de Lyon, Service d'Hépatologie et de Gastroentérologie, Groupement Hospitalier Lyon Nord, F69000 Lyon, France. ${ }^{6}$ Service d'Hématologie, Pavillon Marcel Bérard, Centre Hospitalier Lyon-Sud, Université Lyon I, 165, chemin du Grand Revoyet, 69495 Pierre Bénite, France.

Received: 28 June 2013 Accepted: 22 August 2013

Published: 11 September 2013

\section{References}

1. McGlynn KA, London WT: The global epidemiology of hepatocellular carcinoma: present and future. Clin Liver Dis 2011, 15:223-243. vii-X.

2. Li R, Qian N, Tao K, You N, Wang X, Dou K: MicroRNAs involved in neoplastic transformation of liver cancer stem cells. J Exp Clin Cancer Res 2010, 29:169.

3. Begus-Nahrmann Y, Hartmann D, Kraus J, Eshraghi P, Scheffold A, Grieb M, Rasche V, Schirmacher P, Lee HW, Kestler HA, et al: Transient telomere dysfunction induces chromosomal instability and promotes carcinogenesis. J Clin Invest 2012, 122:2283-2288.

4. Farazi PA, Glickman J, Horner J, Depinho RA: Cooperative interactions of p53 mutation, telomere dysfunction, and chronic liver damage in hepatocellular carcinoma progression. Cancer Res 2006, 66:4766-4773.

5. Farazi PA, Glickman J, Jiang S, Yu A, Rudolph KL, DePinho RA: Differential impact of telomere dysfunction on initiation and progression of hepatocellular carcinoma. Cancer Res 2003, 63:5021-5027.

6. Plentz RR, Caselitz M, Bleck JS, Gebel M, Flemming P, Kubicka S, Manns MP, Rudolph KL: Hepatocellular telomere shortening correlates with chromosomal instability and the development of human hepatoma. Hepatology 2004, 40:80-86.

7. Plentz RR, Park YN, Lechel A, Kim H, Nellessen F, Langkopf BH, Wilkens L, Destro A, Fiamengo B, Manns MP, et al: Telomere shortening and inactivation of cell cycle checkpoints characterize human hepatocarcinogenesis. Hepatology 2007, 45:968-976.

8. Plentz RR, Schlegelberger B, Flemming P, Gebel M, Kreipe H, Manns MP, Rudolph KL, Wilkens L: Telomere shortening correlates with increasing aneuploidy of chromosome 8 in human hepatocellular carcinoma. Hepatology 2005, 42:522-526.

9. Lai XF, Shen CX, Wen Z, Qian YH, Yu CS, Wang JQ, Zhong PN, Wang HL: PinX1 regulation of telomerase activity and apoptosis in nasopharyngeal carcinoma cells. J Exp Clin Cancer Res 2012, 31:12.

10. Bodnar AG, Ouellette M, Frolkis M, Holt SE, Chiu CP, Morin GB, Harley CB, Shay JW, Lichtsteiner S, Wright WE: Extension of life-span by introduction of telomerase into normal human cells. Science 1998, 279:349-352.

11. De Lange T: Shelterin: the protein complex that shapes and safeguards human telomeres. Genes Dev 2005, 19:2100-2110.

12. Gilson E, Geli V: How telomeres are replicated. Nat Rev Mol Cell Biol 2007, 8:825-838.

13. D' Adda Di Fagagna F, Reaper PM, Clay-Farrace L, Fiegler H, Carr P, Von Zglinicki T, Saretzki G, Carter NP, Jackson SP: A DNA damage checkpoint response in telomere-initiated senescence. Nature 2003, 426:194-198.

14. Hayflick $L$ : The limited in vitro lifetime of human diploid cell strains. Exp Cell Res 1965, 37:614-636.

15. Hayflick L, Moorhead PS: The serial cultivation of human diploid cell strains. Exp Cell Res 1961, 25:585-621.

16. Lukas J, Parry D, Aagaard L, Mann DJ, Bartkova J, Strauss M, Peters G, Bartek $\mathrm{J}$ : Retinoblastoma-protein-dependent cell-cycle inhibition by the tumour suppressor p16. Nature 1995, 375:503-506.

17. Stein GH, Drullinger LF, Soulard A, Dulic V: Differential roles for cyclindependent kinase inhibitors p21 and p16 in the mechanisms of senescence and differentiation in human fibroblasts. Mol Cell Biol 1999, 19:2109-2117.

18. Vaziri H, West MD, Allsopp RC, Davison TS, Wu YS, Arrowsmith CH, Poirier GG, Benchimol S: ATM-dependent telomere loss in aging human diploid fibroblasts and DNA damage lead to the post-translational activation of p53 protein involving poly(ADP-ribose) polymerase. The EMBO journal 1997, 16:6018-6033.

19. Artandi SE, Chang S, Lee SL, Alson S, Gottlieb GJ, Chin L, DePinho RA: Telomere dysfunction promotes non-reciprocal translocations and epithelial cancers in mice. Nature 2000, 406:641-645.

20. Artandi SE, DePinho RA: A critical role for telomeres in suppressing and facilitating carcinogenesis. Curr Opin Genet Dev 2000, 10:39-46.

21. Rudolph KL, Millard M, Bosenberg MW, DePinho RA: Telomere dysfunction and evolution of intestinal carcinoma in mice and humans. Nat Genet 2001, 28:155-159.

22. Horikawa I, Barrett JC: Cis-activation of the human telomerase gene (hTERT) by the hepatitis B virus genome. J Natl Cancer Inst 2001, 93:1171-1173.

23. Liu H, Shi W, Luan F, Xu S, Yang F, Sun W, Liu J, Ma C: Hepatitis B virus X protein upregulates transcriptional activation of human telomerase reverse transcriptase. Virus Genes 2010, 40:174-182. 
24. Qu ZL, Zou SQ, Cui NQ, Wu XZ, Qin MF, Kong D, Zhou ZL: Upregulation of human telomerase reverse transcriptase mRNA expression by in vitro transfection of hepatitis $B$ virus $X$ gene into human hepatocarcinoma and cholangiocarcinoma cells. World I Gastroenterol 2005, 11:5627-5632.

25. Su JM, Lai XM, Lan KH, Li CP, Chao Y, Yen SH, Chang FY, Lee SD, Lee WP: X protein of hepatitis $B$ virus functions as a transcriptional corepressor on the human telomerase promoter. Hepatology 2007, 46:402-413.

26. Zhang $X$, Dong $N$, Zhang $H$, You J, Wang $H$, Ye L: Effects of hepatitis $B$ virus $\mathrm{X}$ protein on human telomerase reverse transcriptase expression and activity in hepatoma cells. J Lab Clin Med 2005, 145:98-104.

27. Zou SQ, Qu ZL, Li ZF, Wang X: Hepatitis B virus $X$ gene induces human telomerase reverse transcriptase mRNA expression in cultured normal human cholangiocytes. World J Gastroenterol 2004, 10:2259-2262.

28. Liu H, Luan F, Ju Y, Shen H, Gao L, Wang X, Liu S, Zhang L, Sun W, Ma C: In vitro transfection of the hepatitis $B$ virus PreS2 gene into the human hepatocarcinoma cell line HepG2 induces upregulation of human telomerase reverse transcriptase. Biochem Biophys Res Commun 2007, 355:379-384.

29. Luan F, Liu H, Gao L, Liu J, Sun Z, Ju Y, Hou N, Guo C, Liang X, Zhang L, et al: Hepatitis B virus protein preS2 potentially promotes $\mathrm{HCC}$ development via its transcriptional activation of hTERT. Gut 2009, 58:1528-1537.

30. Zhu Z, Wilson AT, Gopalakrishna K, Brown KE, Luxon BA, Schmidt WN: Hepatitis C virus core protein enhances Telomerase activity in Huh7 cells. J Med Virol 2010, 82:239-248.

31. Pavanello S, Hoxha M, Dioni L, Bertazzi PA, Snenghi R, Nalesso A, Ferrara SD, Montisci M, Baccarelli A: Shortened telomeres in individuals with abuse in alcohol consumption. Int J Cancer 2011, 129:983-992.

32. Pfaffl MW, Tichopad A, Prgomet C, Neuvians TP: Determination of stable housekeeping genes, differentially regulated target genes and sample integrity: bestkeeper-excel-based tool using pair-wise correlations. Biotechnol Lett 2004, 26:509-515.

33. Livak K, Schmittgen TD: Analysis of relative gene expression data using real-time quantitative PCR and the 2(-Delta Delta $C(T))$ Method. Methods 2001, 25:402-408.

34. Saini N, Srinivasan R, Chawla Y, Sharma S, Chakraborti A, Rajwanshi A: Telomerase activity, telomere length and human telomerase reverse transcriptase expression in hepatocellular carcinoma is independent of hepatitis virus status. Liver Int 2009, 29:1162-1170.

35. Guo Y, Zhou X, Liu E, Li X, Liu J, Yang Z, Yi J: Difference in hTERT gene expressions between $\mathrm{HbsAg-positive} \mathrm{and} \mathrm{HbsAg-negative} \mathrm{hepatocellular}$ carcinoma. J Huazhong Univ Sci Technolog Med Sci 2005, 25:303-306.

36. Oh BK, Kim YJ, Park C, Park YN: Up-regulation of telomere-binding proteins, TRF1, TRF2, and TIN2 is related to telomere shortening during human multistep hepatocarcinogenesis. Am J Pathol 2005, 166:73-80.

37. Lazzerini Denchi E, Celli G, De Lange T: Hepatocytes with extensive telomere deprotection and fusion remain viable and regenerate liver mass through endoreduplication. Genes Dev 2006, 20:2648-2653.

38. Hu Y, Shen Y, Ji B, Wang L, Zhang Z, Zhang Y: Combinational RNAi gene therapy of hepatocellular carcinoma by targeting human EGFR and TERT. Eur J Pharm Sci 2011, 42:387-391.

39. Greten TF, Forner A, Korangy F, N'Kontchou G, Barget N, Ayuso C, Ormandy $L A$, Manns MP, Beaugrand $M$, Bruix J: A phase II open label trial evaluating safety and efficacy of a telomerase peptide vaccination in patients with advanced hepatocellular carcinoma. BMC Cancer 2010, 10:209.

40. Guo X, Wang W, Zhou F, Lu Z, Fang R, Jia F, Bu X, Li R, Zhang B, Wu M, Wei L: siRNA-mediated inhibition of hTERT enhances chemosensitivity of hepatocellular carcinoma. Cancer Biol Ther 2008, 7:1555-1560.

\section{Submit your next manuscript to BioMed Central and take full advantage of:}

- Convenient online submission

- Thorough peer review

- No space constraints or color figure charges

- Immediate publication on acceptance

- Inclusion in PubMed, CAS, Scopus and Google Scholar

- Research which is freely available for redistribution

Submit your manuscript at www.biomedcentral.com/submit
C Biomed Central 